\title{
ChemComm
}

CrossMark \& click for updates

Cite this: Chem. Commun., 2015 51,15063

Received 13th August 2015 Accepted 20th August 2015

DOI: $10.1039 / c 5 c c 06819 c$

www.rsc.org/chemcomm

\section{Coordination-directed self-assembly of a simple benzothiadiazole-fused tetrathiafulvalene to low-bandgap metallogels $\dagger$}

\author{
Anneliese M. Amacher, ${ }^{a}$ Josep Puigmartí-Luis, ${ }^{\text {bc }}$ Yan Geng, ${ }^{a}$ Victor Lebedev, ${ }^{b}$ \\ Vladimir Laukhin, ${ }^{\text {bd }}$ Karl Krämer, ${ }^{a}$ Jürg Hauser, ${ }^{a}$ David B. Amabilino, ${ }^{\text {be }}$ \\ Silvio Decurtins ${ }^{a}$ and Shi-Xia Liu*a
}

Coordination-driven gelation of a benzothiadiazole-fused tetrathiafulvalene (TTF) is demonstrated. This is the first work reporting highly stable metallogels based on a donor-acceptor conjugate with such a simple structure for the construction of new low-bandgap materials with various functional properties and novel nanostructures.

Molecular gels find applications in a wide variety of interesting mechanical, electrical, and biological systems. ${ }^{1}$ They can potentially form highly ordered self-assembled structures in a bottom up strategy, facilitating fast and easy fabrication of molecular electronic devices. Their potential application in organic electronics has driven research into new molecular materials containing electron donors, acceptors, or combined donor-acceptor moieties. The interaction between donor and acceptor allows the specification and control of energy levels, critical aspects that dramatically influence the performance. $^{2}$ There has been a relative dearth, however, of donor-acceptor compounds incorporated into metallogels that assemble through $\pi-\pi$ stacking or metal-ligand interactions. ${ }^{3}$ Depending on the metal centres, coordination geometry and coordinating ligands, metallogels exhibit intriguing spectroscopic, catalytic and redox properties. ${ }^{3,4}$

Our interest in both electron donor-acceptor materials and supramolecular systems motivated us to explore the possibility of supramolecular organization created from a benzothiadiazole (BTD)-fused tetrathiafulvalene (TTF), as shown in Fig. 1. To our

\footnotetext{
${ }^{a}$ Departement für Chemie und Biochemie, Universität Bern, Freiestrasse 3, CH-3012 Bern, Switzerland. E-mail: liu@dcb.unibe.ch

${ }^{b}$ Institut de Ciència de Materials de Barcelona (ICMAB-CSIC), Campus Universitari de Bellaterra, 08193, Catalonia, Spain

${ }^{c}$ Empa, Laboratory for Protection and Physiology, Lerchenfeldstrasse 5, CH-9014 St. Gallen, Switzerland

${ }^{d}$ ICREA, Barcelona, Spain

${ }^{e}$ School of Chemistry, The University of Nottingham, University Park, Nottingham, NG7 2RD, UK. E-mail: David.Amabilino@nottingham.ac.uk

$\dagger$ Electronic supplementary information (ESI) available: Single crystal structure of 1, IR and NMR spectra, temperature-dependent powder X-ray diffraction measurements, thermal analysis, magnetic susceptibility measurement, SEM images and EPR measurements. CCDC 1005516. For ESI and crystallographic data in CIF or other electronic format see DOI: $10.1039 / \mathrm{c} 5 \mathrm{cc} 06819 \mathrm{c}$
}

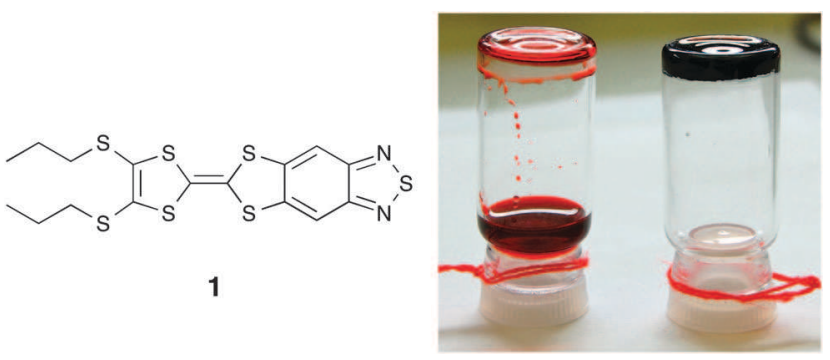

Fig. 1 (left) Chemical structure of the gelator 1; (right) photograph showing, in the left vial, the control experiment of $\mathbf{1}$ in chloroform and ethanol with no metal salt added and in the right vial, upon addition of $\mathrm{CuCl}_{2}$.

surprise, we found that $\mathbf{1}$ acts as a low molecular weight gelator (LMWG) in the presence of certain transition metal halide salts. This self-assembly process is mainly driven by the coordination ability of the BTD moiety to metal ions. ${ }^{5}$ Although TTF-based $\mathrm{LMWGs}^{6,7}$ as well as supramolecular self-assembled structures of TTF ligands with some transition metal ions ${ }^{8}$ have been reported in the literature, their metallogels are not known. On the other hand, all of the reported TTF gelators have specific structural characteristics that are frequently employed to drive gelation; for example, the inclusion of amide or other hydrogenbonding moieties as well as long fatty chains to increase van der Waals interactions. The present work represents the first example of low-bandgap metallogels based on a TTF gelator with a very simple structure. We describe various microscopic and spectroscopic techniques used to characterize these metallogels, which are both more pure and more facile to synthesize than the metallopolymers formed through covalent bonds.

The gelator 1 was obtained by a phosphite-mediated crosscoupling reaction of 4,5-bis(propylsulfanyl)-1,3-dithiol-2-thione and [1,3]dithiolo[4,5-f]-2,1,3-benzothiadiazole-6-one ${ }^{9}$ in $61 \%$ yield. It complexes with $\mathrm{MX}_{2}\left(\mathrm{M}(\mathrm{II})=\mathrm{Cu}, \mathrm{Cd}, \mathrm{Co} ; \mathrm{X}=\mathrm{Cl}^{-}\right.$and $\left.\mathrm{Br}^{-}\right)$to gel solvents forming multi-component supramolecular metallopolymers. As illustrated in Fig. 1, gelation occurred in less than five minutes upon mixing an ethanol solution of copper chloride ( 1 equiv.) to a solution of $\mathbf{1}$ in $\mathrm{CHCl}_{3}$; the gel is very stable to 
inversion and is self-sustaining. Upon gelation, a dramatic colour change from clear red to dark blue was observed. The effect of solvent on gelation was tested. The critical gel concentration of 1 in different solvents when combined with $\mathrm{CuCl}_{2}$ in ethanol is as follows: chloroform, $0.02 \mathrm{M}$; dichloromethane, $0.04 \mathrm{M}$; chlorobenzene, $0.03 \mathrm{M}$; and $o$-dichlorobenzene, $0.04 \mathrm{M}$. The gelation was not effective in non-halogenated solvents.

To further explore the role of metal salts, different metal cations (Table S1, ESI $\dagger$ ) and counterions (Table S2, ESI $\dagger$ ) were chosen for the gelation studies. The result was that $\mathrm{MX}_{2}$ with $\mathrm{M}(\mathrm{II})=\mathrm{Cu}, \mathrm{Cd}, \mathrm{Co}\left(\mathrm{X}=\mathrm{Cl}^{-}, \mathrm{Br}^{-}\right)$can form dark blue gels, but not in the cases of $\mathrm{Zn}$ (II), $\mathrm{Mn}$ (II) and Fe(II). The anion clearly plays a role in the gelation, as fluoride and perchlorate copper salts do not form gels. The halide ions show high propensity to form gels, very probably ascribed to their ability to form metal clusters via halide bridges, ${ }^{10}$ and here we focus on these salts.

It has to be noted that the metallogels exhibit high stability as evidenced by the fact that the sol-gel transformation cannot be tuned by external chemical stimuli. For example, differential scanning calorimetry (DSC) measurements of the $\mathrm{CuCl}_{2}$ xerogel (Fig. S1, ESI $\dagger$ ) indicate three irreversible endothermic transitions, very probably due to the loss of solvents and the solid-state-like phase transition. It appears that the gel is thermo-irreversible as it remains intact when heated up to $70{ }^{\circ} \mathrm{C}$ while it is gradually fragmented upon increasing the temperature, finally leading to a dark blue precipitate in solution. It is neither responsive to a metal trapping reagent - such as ethylene diamine tetraacetic acid (EDTA) - nor to oxidation. Additionally, the reversibility of the gelation process did not occur through mechanical stimuli either. When shaking breaks a gel, the appearance of the system becomes one that is composed of fibres dispersed in a solvent. This effect is irreversible. All these results demonstrate that metallogels possess a rigid rather than flexible suprastructure.

The UV-visible absorption spectra of 1 in a $\mathrm{CHCl}_{3}$ solution and as a film as well as of a $\mathrm{CuCl}_{2}$ gel are shown in Fig. 2 . According to our previous results of analogues, ${ }^{11}$ the intense absorption band peak at $488 \mathrm{~nm}$ in solution corresponds to an intramolecular charge-transfer transition from the HOMO localized on the TTF core to the LUMO localized on the BTD moiety. A slight

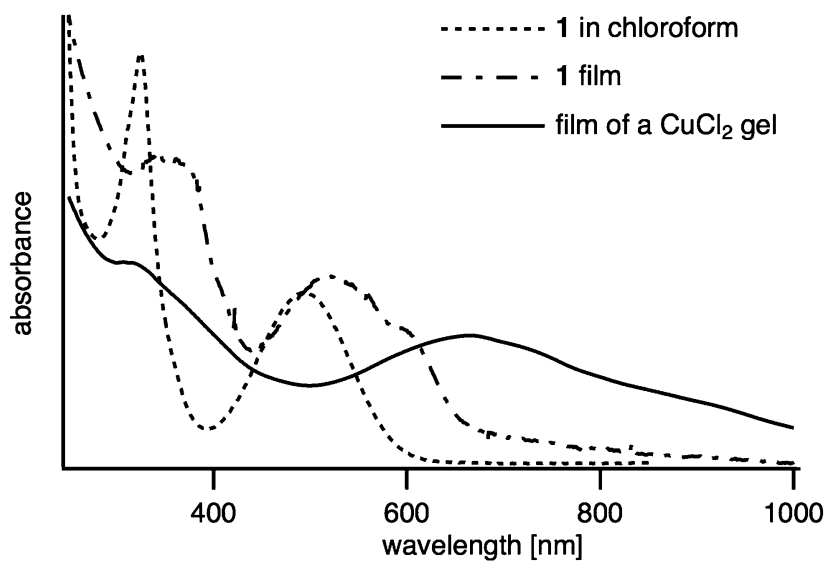

Fig. 2 UV-vis spectra of 1 in solution, film, and as a $\mathrm{CuCl}_{2}$ gel. red-shift of this optical absorbance in the film is attributed to strong intermolecular interactions. The copper chloride gel shows an even larger red-shift extending into the near-IR region giving rise to a low bandgap $(<1.2 \mathrm{eV})$, indicative of the coordination of $\mathrm{Cu}$ (II) by the nitrogen atoms on the BTD portion of 1 . These results are consistent with the ${ }^{1} \mathrm{H}$ NMR data of the $\mathrm{CdBr}_{2}$ gel (Fig. S2, ESI $\dagger$ ). Upon gelation, the signals due to the benzene ring and propyl groups of $\mathbf{1}$ become broad and are shifted upfield. Additionally, FTIR spectra of $\mathbf{1}$ and its $\mathrm{MCl}_{2}$ xerogels (Fig. S3, ESI $\dagger$ ) indicate slight shifts in the vibration frequencies for the BTD aromatic ring in the range of $1200 \mathrm{~cm}^{-1}$ and $1500 \mathrm{~cm}^{-1}$ upon gelation. All these observations imply that the coordination bonds are primary driving forces to form the fibers while the $\pi-\pi$ stacking between BTD-fused TTF units and noncovalent van der Waals interactions play an important role in the entanglement of fibers and the capture of the solvent.

Powder X-ray diffraction patterns of a xerogel from $\mathrm{CuCl}_{2}$ and $\mathbf{1}$ as well as of monomer 1 on its own are shown in Fig. S4 (ESI $\dagger$ ). The gel shows some amorphous as well as crystalline character, as evidenced by the presence of both broad and sharp peaks. The gel spectrum exhibits some similarity to $\mathbf{1}$ but also new features that do not correspond to either pure $\mathrm{CuCl}_{2}$ or $\mathbf{1}$, indicating that the self-organization in the gel is not simply a precipitated monomer, but a new hybrid of both metal ions and $\mathbf{1}$.

Magnetic susceptibility (SQUID) measurements (Fig. S5, ESI $\dagger$ ) also determined that there was $8.3 \%$ of copper(II) in the xerogel, which is close to the expected value of $11 \%$ for a $1: 1$ ratio of 1 to copper(II) in the bulk gel. Moreover, the magnetic susceptibility data show that there is a pronounced antiferromagnetic coupling between neighbouring $\mathrm{Cu}$ (II) ions. These results indicate that $\mathrm{Cu}$ (II) is involved in the gelation and not just a precipitate dispersed in the gel matrix.

Scanning electron microscopy (SEM) was used to visualize the morphology of the xerogels formed under different conditions. As depicted in Fig. S6 (ESI $\dagger$ ), the gels have a wavy suprastructure in less polar solvents, such as chloroform and chlorobenzene, and more flat structures in more polar solvents, such as $o$-dichlorobenzene and dichloromethane. The 3-D network has a woven appearance with belt-like fibers of about $50 \mathrm{~nm}$ in diameter, which shows some alignment over several tens of microns. Similar features, albeit with some subtle differences, were observed in the SEM images of other metallogels (Fig. S7, ESI $\dagger$ ).

The gels show an intriguing response to washing (Fig. S8, ESI $\dagger$ ). The material washed away depends upon the washing solvent. The dark blue xerogel is initially rinsed with ethanol, which washes away the metal salt and changes the colour to that of 1, red. This observation can be confirmed by energydispersive X-ray spectroscopy (EDX). For instance, the atomic ratio of $\mathrm{S}: \mathrm{Cu}$ changes from 70:10 for the as synthesized gel to $96: 2$ for the gel washed with ethanol. Also SEM images reveal the variation of the fibrous nature of the gel by washing (Fig. S9, ESI $\dagger$ ). The as synthesized gel shows a columnar structure with overlying parallel wavy features. When the gel is washed with ethanol, however, what remains appears very similar to pure $\mathbf{1}$, suggesting the removal of $\mathrm{CuCl}_{2}$. When the gel is further rinsed with dichloromethane, the layers of $\mathbf{1}$ are removed from the gel surface, and the gel turns dark blue. This process of selectively 
washing away portions of the gel by alternating solvents is indicative of the formation of a stable layered suprastructure (Fig. S10, ESI $\dagger$ ) with a lamellar structural sequence during the gelation process. This structural feature is not beneficial to the penetration of the solid EDTA into the gel, which accounts for the aforementioned failure of EDTA to uptake $\mathrm{Cu}(\mathrm{II})$ from the gel.

While bulk gels are interesting, electronically active materials require easy processability into thin films for useful device manufacture. To that end effort was focused on creating homogeneous thin films. Ideally, spin coating or drying over a thermal gradient would be a favourable method for it, however, due to the gel-like consistency of the material we probed another route. Loose gels, made from a mixture of a solution of $\mathbf{1}$ in $o$-dichlorobenzene and a solution of $\mathrm{CuCl}_{2}$ in ethanol, were sandwiched between two microscope slides, which were then pressed together to blend the green gelatinous particulates (visible under an optical microscope) into an amorphous green film. The glass slides were then slid laterally apart. The resulting films were allowed to dry, whereupon they darkened. When viewed under a polarizing optical microscope, a homogeneous film of uniform alignment responded to the polarized light, indicating the parallel alignment of the chromophoric units. A SEM image of the resulting film (Fig. S11, ESI $\dagger$ ) shows that there are features on the scale of $500 \mathrm{~nm}$ to $1 \mu \mathrm{m}$.

A doped thin film of the $\mathrm{CuCl}_{2}$ xerogel was prepared after exposure to iodine vapors for two minutes and further investigated as a conductor. Electron paramagnetic resonance (EPR) spectroscopy is useful for exploring the electronic characteristics of this kind of material. ${ }^{6 d}$ As illustrated in Fig. S12 (ESI $\dagger$ ), the un-doped xerogel is EPR signal silent whereas the doped one exhibits a clear EPR signal with a peak-to-peak linewidth $(\triangle \mathrm{Hpp})$ of $30 \mathrm{G}$. This EPR signal corresponds to the formation of the cation radical of $1 .{ }^{12}$ Surprisingly, when the doped xerogels were subjected to an annealing cycle, an irreversible decrease of the peak-to-peak linewidth was observed from $30 \mathrm{G}$ to $6 \mathrm{G}$. During the annealing process (Fig. S13, ESI $\dagger$ ), the samples were heated up to $200{ }^{\circ} \mathrm{C}$ and subsequently cooled down to room temperature. This change in $\Delta \mathrm{Hpp}$ can be attributed to an irreversible solid-state-like transition to a new structural phase, as reported previously. ${ }^{6 d}$ This phase transition was observed when doped samples were heated up to $200{ }^{\circ} \mathrm{C}$. Interestingly, a peak-topeak linewidth of $6 \mathrm{G}$ is characteristic of oxidized TTF-based organic conductors. ${ }^{13}$ Therefore, four-probe DC resistance measurements on doped $\mathrm{CuCl}_{2}$ xerogels were carried out to study the electrical properties of the two phases and, furthermore, to control the change in the electrical properties during the phase transition. In a typical experiment, a freshly doped xerogel sample was introduced into an oven that was previously set at $200{ }^{\circ} \mathrm{C}$ while a four-probe DC measurement of the sample resistance was conducted at the same time. Clearly, we observed typical semiconductor behaviour in the doped xerogel sample, as the resistance decreased with increasing temperature (Fig. 3) ${ }^{6 d, 14}$ However, around $150{ }^{\circ} \mathrm{C}$, a change in the slope of the resistance versus temperature curve was observed, hence underpinning the phase transition studied using EPR spectroscopy. Also, the low angle X-ray powder diffraction patterns of undoped and doped $\mathrm{Cu}$ xerogels as a function of temperature

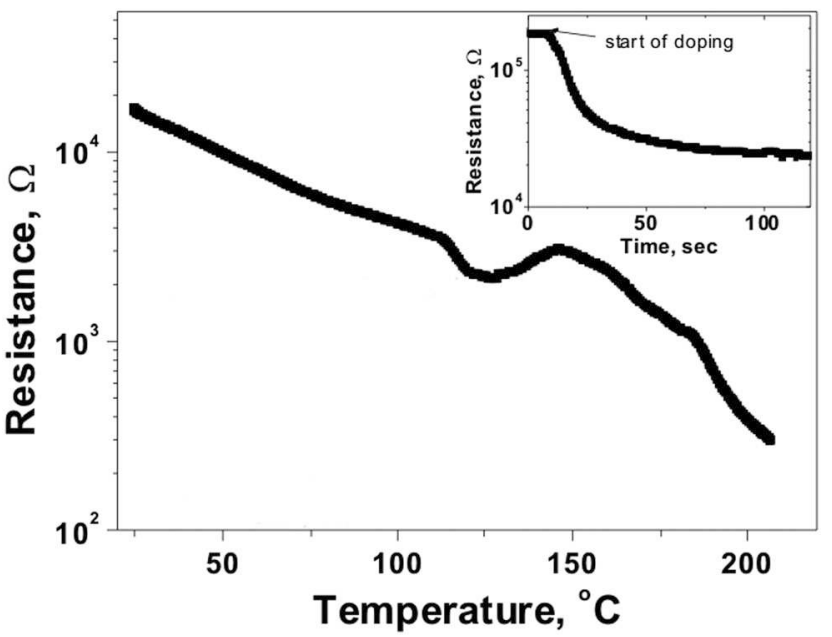

Fig. 3 (a) Temperature dependence of the resistance for a doped $\mathrm{CuCl}_{2}$ xerogel of 1 during annealing (main panel). Top inset shows typical dependence of resistance vs. time during doping by iodine vapor at room temperature.

(Fig. S14, ESI $\dagger$ ) clearly indicate different phase transitions between $100{ }^{\circ} \mathrm{C}$ and $175^{\circ} \mathrm{C}$. Even though the room temperature resistivity is practically the same for the two phases (around $1.8 \pm 0.2 \Omega \mathrm{cm}$ ), their topography changed considerably indicating the irreversible nature of the phase transition (Fig. S15, ESI $\dagger$ ). While smooth wire-like morphologies were observed for freshly doped xerogel samples, needle-like structures appeared after an annealing cycle was conducted. This change in morphology clearly emphasizes the effect of temperature on the metallo-supramolecular organization.

The electrical properties of the doped film of the $\mathrm{CuCl}_{2}$ xerogel of $\mathbf{1}$ were also studied by current sensing atomic force microscopy (CS-AFM). Typical images show regions of high conductivity that have an acicular shape (Fig. 4). These features do not necessarily coincide with the points of greatest height in the film of the xerogel, a feature that rules out any topographic anomaly. In general, scaly features can be appreciated in the topography and the conducting regions are approximately $300 \mathrm{~nm}$ wide and up to a micron long, although it is clear that the bulk material is a conductor, otherwise no imaging would be possible. (The contact is made through the graphite substrate.) The $I / V$ sweeps at different locations show the clear presence of a gap, characteristic of a semiconducting material.

In summary, we have investigated gelation behaviour of a simple BTD-fused TTF primarily through coordination bonds of $\mathrm{MX}_{2}\left(\mathrm{M}(\mathrm{II})=\mathrm{Cu}, \mathrm{Cd}, \mathrm{Co} ; \mathrm{X}=\mathrm{Cl}^{-}\right.$and $\left.\mathrm{Br}^{-}\right)$with the BTD moiety. Among the tested transition metal ions, $\mathrm{Cu}(\mathrm{II})$ is most likely to undergo self-sustaining gelation. The morphologies of the metallogels depend upon the solvent used to dissolve $\mathbf{1}$. Furthermore, EPR and four-probe DC resistance measurements confirm that these doped $\mathrm{CuCl}_{2}$ xerogels can undergo a hightemperature phase transition to a new phase with similar electronic properties to the as-prepared conducting xerogels. This is the first work reporting TTF-based metallogels and opens up an avenue for the construction of new low-bandgap materials with various functional properties and novel nanostructures. It is 

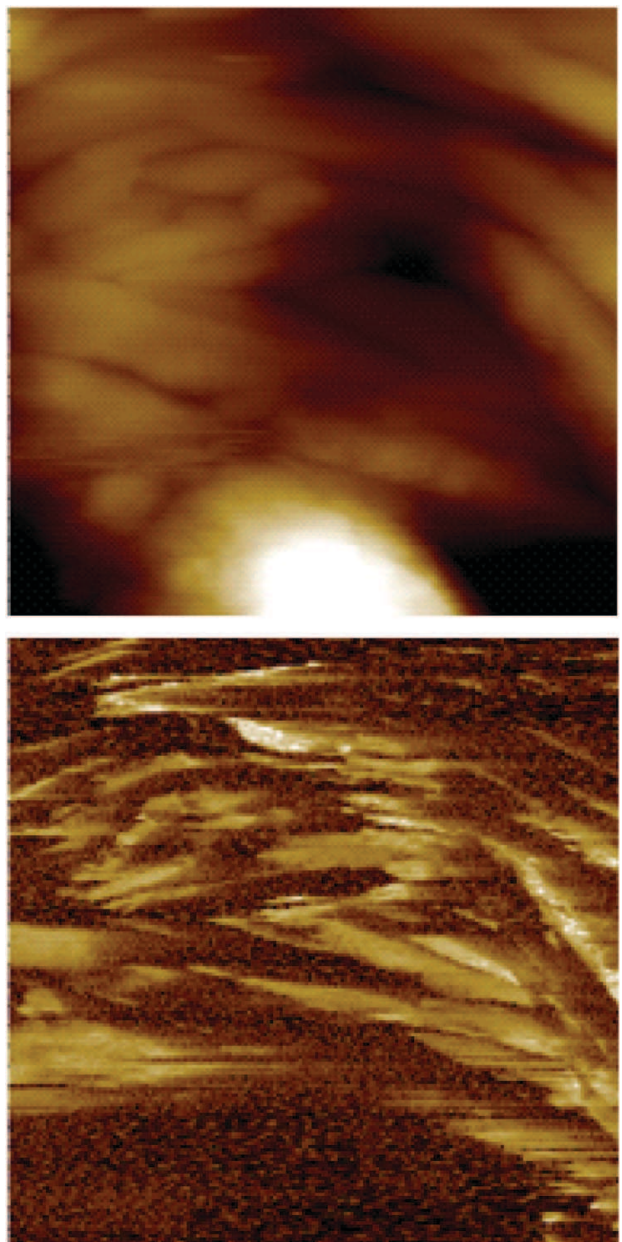

Fig. 4 Topography (top) and current (bottom, bias voltage $1.1 \mathrm{~V}$ ) images from CS-AFM of the same region ( $3 \times 3$ microns) of a $\mathrm{CuCl}_{2}$ xerogel of 1 on highly oriented pyrolytic graphite. The brighter regions in the current map correspond to higher conductivity. The independence of the responses is highlighted by the insulating bump in the topographic image.

envisaged that such intensely coloured metallopolymers represent a promising class of materials for photovoltaic applications.

This work was supported by the Swiss National Science Foundation (No. 200021-147143). JPL thanks MINECO for a Ramón y Cajal contract (RYC-2011-08071); DBA thanks MINECO, Spain (project CTQ2010-16339).

\section{Notes and references}

1 Functional Molecular Gels, ed. B. Escuder and J. F. Miravet, Cambridge, Royal Society of Chemistry (RSC Soft Matter Series), 2014.

2 (a) Y. Matsuo, M. Maruyama, S. S. Gayathri, T. Uchida, D. M. Guldi, H. Kishida, A. Nakamura and E. Nakamura, J. Am. Chem. Soc., 2009,
131, 12643; (b) J. M. Lehn, Proc. Natl. Acad. Sci. U. S. A., 2002, 99, 4763; (c) J. Roncali, Chem. Rev., 1997, 97, 173.

3 (a) C. Qin, Y. Fu, C.-H. Chui, C.-W. Kan, Z. Xie, L. Wang and W.-Y. Wong, Macromol. Rapid Commun., 2011, 32, 1472; (b) C. Jia, S.-X. Liu, C. Ambrus, A. Neels, G. Labat and S. Decurtins, Inorg. Chem., 2006, 45, 3152; (c) W.-Y. Wong, X.-Zh. Wang, Z. He, K.-K. Chan, A. B. Djurišić, K.-Y. Cheung, C.-T. Yip, A. Man-Ching Ng, Y. Y. Xi, C. S. K. Mak and W.-K. Chan, J. Am. Chem. Soc., 2007, 129, 14372.

4 (a) W. Y. Wong and C. L. Ho, Acc. Chem. Res., 2010, 43, 1246; (b) A. Y. Tam and V. W. Yam, Chem. Soc. Rev., 2013, 42, 1540; (c) I. Imaz, M. Rubio-Martínez, W. J. Saletra, D. B. Amabilino and D. Maspoch, J. Am. Chem. Soc., 2009, 131, 18222.

5 (a) G. S. Papaefstathiou, A. Tsohos, C. P. Raptopoulou, A. Terzis, V. Psycharis, D. Gatteschi and S. P. Perlepes, Cryst. Growth Des., 2001, 1, 191; (b) G. S. Papaefstathiou, S. P. Perlepes, A. Escuer, R. Vicente, A. Gantis, C. P. Raptopoulou, A. Tsohos; V. Psycharis, A. Terzis and E. G. Bakalbassis, J. Solid State Chem., 2001, 159, 371.

6 (a) T. Kitahara, M. Shirakawa, S.-I. Kawano, U. Beginn, N. Fujita and S. Shinkai, J. Am. Chem. Soc., 2005, 127, 14980; (b) C. Wang, D. Zhang and D. Zhu, J. Am. Chem. Soc., 2005, 127, 16372; (c) D. Canevet, A. Perez del Pino, D. B. Amabilino and M. Salle, Nanoscale, 2011, 3, 2898; (d) J. Puigmarti-Luis, V. Laukhin, A. Perez del Pino, J. VidalGancedo, C. Rovira, E. Laukhina and D. B. Amabilino, Angew. Chem., Int. Ed., 2007, 46, 238.

7 (a) C. Wang, Q. Chen, F. Sun, D. Zhang, G. Zhang, Y. Huang, R. Zhao and D. Zhu, J. Am. Chem. Soc., 2010, 132, 3092; (b) J. Puigmarti-Luis, A. Perez del Pino, V. Laukhin, L. N. Feldborg, C. Rovira, E. Laukhina and D. B. Amabilino, J. Mater. Chem., 2010, 20, 466.

8 (a) B. Chen, Z.-P. Lv, C. F. Leong, Y. Zhao, D. M. D'Alessandro and J.-L. Zuo, Cryst. Growth Des., 2015, 15, 1861; (b) Y.-D. Huang, P. Huo, M.-Y. Shao, J.-X. Yin, W.-C. Shen, Q.-Y. Zhu and J. Dai, Inorg. Chem., 2014, 53, 3480; (c) Y. Qiao, Y. Lin, S. Liu, S. Zhang, H. Chen, Y. Wang, Y. Yan, X. Guo and J. Huang, Chem. Commun., 2013, 49, 704; (d) S. Bivaud, J.-Y. Balandier, M. Chas, M. Allain, S. Goeb and M. Salle, J. Am. Chem. Soc., 2012, 134, 11968.

9 Y. Geng, R. Pfattner, A. Campos, J. Hauser, V. Laukhin, J. Puigdollers, J. Veciana, M. Mas-Torrent, C. Rovira, S. Decurtins and S.-X. Liu, Chem. - Eur. J., 2014, 20, 7136.

10 (a) U. Englert, Coord. Chem. Rev., 2010, 254, 537; (b) S. Samai and K. Biradha, Chem. Mater., 2012, 24, 1165.

11 (a) C.-Y. Jia, S.-X. Liu, C. Tanner, C. Leiggener, A. Neels, L. Sanguinet, E. Levillain, S. Leutwyler, A. Hauser and S. Decurtins, Chem. - Eur. J., 2007, 13, 3804; (b) Y. Geng, C. Fiolka, K. Krämer, J. Hauser, V. Laukhin, S. Decurtins and S.-X. Liu, New J. Chem., 2014, 38, 2052; (c) F. Pop, A. Amacher, N. Avarvari, J. Ding, L. M. Lawson Daku, A. Hauser, M. Koch, J. Hauser, S.-X. Liu and S. Decurtins, Chem. - Eur. J., 2013, 19, 2504; (d) H.-P. Jia, J. Ding, Y.-F. Ran, S.-X. Liu, C. Blum, I. Petkova, A. Hauser and S. Decurtins, Chem. - Asian J., 2011, 6, 3312; (e) A. Amacher, H. Luo, Z. Liu, M. Bircher, M. Cascella, J. Hauser, S. Decurtins, D. Zhang and S.-X. Liu, RSC Adv., 2014, 4, 2873.

12 E. E. Laukhina, V. A. Merzhanov, S. I. Pesotskii, A. G. Khomenko, E. B. Yagubskii, J. Ulanski, M. Kryszewski and J. K. Jeszka, Synth. Met., 1995, 70, 797.

13 (a) J. Puigmarti-Luis, A. Pérez del Pino, E. Laukhina, J. Esquena, V. Laukhin, C. Rovira, J. Vidal-Gancedo, A. G. Kanaras, R. J. Nichols, M. Brust and D. B. Amabilino, Angew. Chem., Int. Ed., 2008, 47, 1861; (b) J. Puigmarti-Luis, E. Laukhina, V. N. Laukhin, A. Pérez del Pino, N. Mestres, J. Vidal-Gancedo, C. Rovira and D. B. Amabilino, Adv. Funct. Mater., 2009, 19, 934; (c) Y. Tomkiewicz and A. R. Taranko, Phys. Rev. B: Condens. Matter Mater. Phys., 1978, 18, 733; (d) R. B. Somoano, A. Gupta, V. Hadek, T. Datta, M. Jones, R. Deck and A. M. Hermann, J. Chem. Phys., 1975, 63, 4970.

14 M. Luo, T. Ishida, T. Nogami and A. Kobayashi, Synth. Met., 1998, 96, 97. 